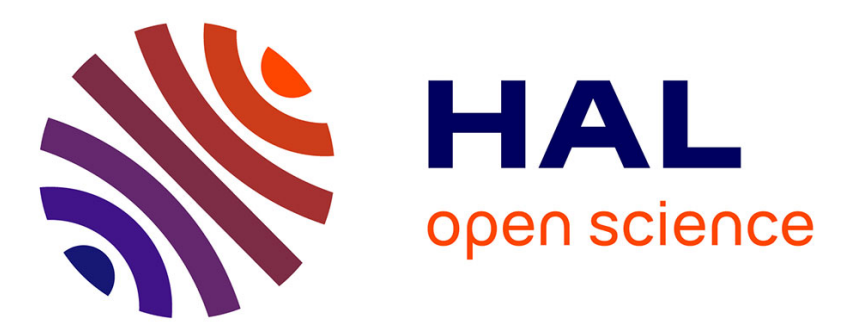

\title{
Deux procédés utilisables pour obtenir une correction de dilatation avec des cavités résonnantes en hyperfréquence
}

\author{
A. Poinsot, S. Sliwa, G. Vosgien
}

\section{- To cite this version:}

A. Poinsot, S. Sliwa, G. Vosgien. Deux procédés utilisables pour obtenir une correction de dilatation avec des cavités résonnantes en hyperfréquence. Revue de Physique Appliquée, 1977, 12 (4), pp.611614. 10.1051/rphysap:01977001204061100 . jpa-00244216

\section{HAL Id: jpa-00244216 https://hal.science/jpa-00244216}

Submitted on 1 Jan 1977

HAL is a multi-disciplinary open access archive for the deposit and dissemination of scientific research documents, whether they are published or not. The documents may come from teaching and research institutions in France or abroad, or from public or private research centers.
L'archive ouverte pluridisciplinaire HAL, est destinée au dépôt et à la diffusion de documents scientifiques de niveau recherche, publiés ou non, émanant des établissements d'enseignement et de recherche français ou étrangers, des laboratoires publics ou privés. 
Classification

Physics Abstracts

$0.660-8.700$

\title{
DEUX PROCÉDÉS UTILISABLES POUR OBTENIR UNE CORRECTION DE DILATATION AVEC DES CAVITÉS RÉSONNANTES EN HYPERFRÉQUENCE
}

\author{
A. POINSOT, S. SLIWA et G. VOSGIEN \\ Laboratoire de Radioélectricité, Université de Dijon, France
}

(Reçu le 9 novembre 1976, accepté le 21 décembre 1976)

\begin{abstract}
Résumé. - Les cavités résonnantes utilisées en hyperfréquence pour des caractérisations de matériaux diélectriques présentent, lors d'études en fonction de la température, une dérive non négligeable de leur fréquence de résonance. Nous présentons les résultats obtenus avec deux procédés de correction. Le premier consiste à stabiliser la fréquence de résonance elle-même par le jeu des dilatations différentielles de deux métaux différents.

Le second, plus général, utilise la stabilisation du battement entre les fréquences propres de deux cavités identiques placées dans les mêmes conditions de température. Dans les deux cas on obtient une dérive résiduelle de l'ordre de $1 \mathrm{kHz} /{ }^{\circ} \mathrm{C}$ pour des températures variant de $-50^{\circ} \mathrm{C}$ à $+100^{\circ} \mathrm{C}$.
\end{abstract}

\begin{abstract}
Microwave resonant cavities used for the characterization of dielectric materials have a non negligible intrinsic drift of their resonant frequency in experiments where the temperature is a variable parameter. We give results obtained with two methods of correction. The first method consists in stabilizing the resonant frequency itself by using the differential expansion of two different metals. The second method relies on stabilizing the beat frequency between the eigenfrequencies of two identical cavities at the same temperature. In both cases we obtain a residual drift near $1 \mathrm{kHz} /{ }^{\circ} \mathrm{C}$ for temperatures between $-50^{\circ} \mathrm{C}$ and $+100{ }^{\circ} \mathrm{C}$.
\end{abstract}

1. Introduction. - Les cavités résonnantes constituent un moyen très utilisé pour la caractérisation des matériaux en hyperfréquence. Lorsqu'on veut étudier le comportement d'un matériau en fonction de la température, on est conduit à chauffer la cavité contenant l'échantillon. Les paramètres propres de la cavité, principalement sa fréquence de résonance, varient aussi avec la température, ce qui a deux effets désagréables. D'abord il faut faire, à chaque température, les corrections appropriées sur les mesures, ensuite il est possible qu'une faible perturbation de la fréquence de résonance par l'échantillon, soit masquée par une dérive plus importante due à la dilatation propre de la cavité.

Nous étudions ici deux méthodes, que nous avons expérimentées et qui permettent, avec une précision suffisante, d'ignorer la dilatation propre de la cavité lors de mesures de constantes diélectriques en fonction de la température. La première consiste à obtenir une compensation de la fréquence de résonance par le jeu des dilatations différentielles de deux métaux différents. La seconde consiste à construire deux cavités identiques dont on compare les fréquences de résonance. Cette comparaison peut se faire facilement par battement.
2. Cavité compensée. - Cette cavité a été construite en vue d'études de substrats utilisés dans les microcircuits hyperfréquences. Garault, Guillon et Jallagéas ont proposé une méthode sensible et non destructive [1] qu'ils ont appliquée à des mesures de $\varepsilon^{\prime}$ et $\operatorname{tg} \delta$ à la température ambiante. Nous nous sommes proposé d'étendre les mesures à différentes températures [2].

La cavité cylindrique utilisée résonne sur le mode TE 013 au voisinage de $9000 \mathrm{MHz}$. L'échantillon, sous forme d'une plaquette, de dimensions standardisées, $50,80 \times 50,80 \times 0,635 \mathrm{~mm}^{3}$, est placé à mihauteur de la cavité, dont il couvre entièrement la section droite. Les lignes de champ électrique et les lignes de courant dans les parois sont des cercles admettant tous le même axe. De ce fait il est possible de couper la cavité selon n'importe quel plan de section droite, ce qui autorise une réalisation mécanique particulièrement simple (Fig. 1). En effet la cavité peut être assemblée en 4 parties : deux manchons cylindriques ( 1 et 2 ) et deux couvercles (3 et 4). Un logement pour l'échantillon est ménagé au raccordement des deux manchons cylindriques. Des pièges quartd'onde creusés dans les couvercles permettent d'atténuer fortement le mode compagnon TM 113. 


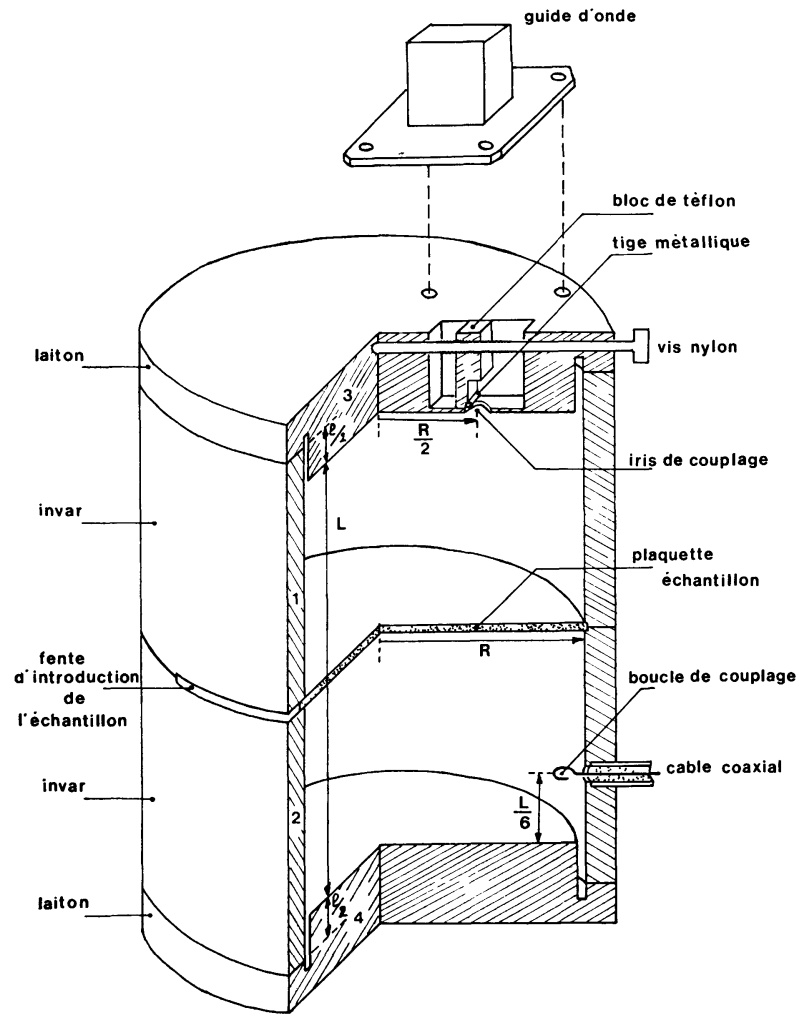

Fig. 1. - Vue générale de la cavité compensée (Mode TE 013).

[General view of the compensated cavity (Mode TE 013)]

Dans une première réalisation les couplages étaient assurés par deux boucles, disposées dans des plans de section droite à $1 / 6$ ou $5 / 6$ de la hauteur, et alimentées par des câbles coaxiaux semi-rigides.

A l'usage il s'est avéré qu'un couplage par iris, sur une face plane [3], présentait une meilleure stabilité en température. Le guide d'onde est orienté de façon à ce que le champ électrique dans le guide soit tangent aux lignes de champ électrique circulaires dans la cavité. L'iris est placé au niveau d'un ventre de $E_{\theta}$, soit à une distance de l'axe égale environ au demi-rayon de la cavité. Le coefficient de couplage peut être ajusté à la valeur désirée par déplacement d'une tige métallique devant l'iris. Cette tige, de diamètre $1 \mathrm{~mm}$, est portée par un bloc de téflon situé dans le guide. Sur la figure 1 , ce bloc de téflon a été découpé de façon à faire apparaître la position de la tige, sur le fond du guide, et son orientation, parallèle au champ électrique dans le guide.

Le corps cylindrique de la cavité a été construit en invar, ce qui assure une bonne stabilité du diamètre avec la température. Un dépôt électrolytique d'argent est nécessaire pour améliorer la conductivité des parois. Les couvercles réentrants sont en laiton, qui se dilate environ 20 fois plus que l'invar.

La fréquence de résonance $f$ est obtenue à partir du diamètre $D$ de la cavité et de sa hauteur $L$ par

$$
f^{2}=\frac{A}{D^{2}}+\frac{B}{L^{2}}
$$

où $A$ et $B$ sont deux constantes caractéristiques du mode de résonance. Si $l$ est la longueur de la partie réentrante en laiton, on aura, à la température $\theta$,

$$
\begin{aligned}
& D=D_{0}\left(1+\alpha_{\mathrm{I}} \theta\right) \\
& L=\left(L_{0}+l_{0}\right)\left(1+\alpha_{\mathrm{I}} \theta\right)-l_{0}\left(1+\alpha_{\mathrm{L}} \theta\right)
\end{aligned}
$$

$\alpha_{\mathrm{L}}$ étant le coefficient de dilatation du laiton, $\alpha_{\mathrm{I}}$ celui de l'invar. $\mathrm{Si}$

$$
l_{0}>\frac{\alpha_{I}}{\alpha_{L}-\alpha_{I}} L_{0},
$$

une augmentation de température se traduit par une augmentation de diamètre et une diminution de hauteur de volume résonnant. Si $l_{0}$ est convenablement ajusté ces deux effets peuvent se compenser pour maintenir constante la fréquence de résonance. Un calcul au premier ordre donne :

$$
l_{0}=\frac{\alpha_{\mathrm{I}}}{\alpha_{\mathrm{L}}-\alpha_{\mathrm{I}}} L_{0}\left(1+\frac{A}{B} \frac{L_{0}^{2}}{D_{0}^{2}}\right) .
$$

Dans notre réalisation

$$
D_{0}=50,5 \mathrm{~mm}, L_{0}=84,1 \mathrm{~mm} \text { et } l_{0}=11,9 \mathrm{~mm} \text {. }
$$

L'étude expérimentale de la fréquence de résonance avec la température, à l'aide d'un fréquencemètre à haute résolution, a donné les résultats suivants:

- entre $-50^{\circ} \mathrm{C}$ et $+70^{\circ} \mathrm{C}$, la fréquence se maintient dans une plage de largeur $25 \mathrm{kHz}$;

- au-delà de $70{ }^{\circ} \mathrm{C}$ on voit apparaître la nonlinéarité et la fréquence décroît de plus en plus vite. Toutefois la pente de la sécante $0-100^{\circ} \mathrm{C}$ n'est que

$$
\frac{\Delta f}{\Delta \theta}=-1,2 \mathrm{kHz} /{ }^{\circ} \mathrm{C} \text {. }
$$

Il est intéressant de comparer ce résultat avec les valeurs théoriques obtenues pour une cavité non compensée :

- cavité tout invar : $\Delta f / \Delta \theta=-8,1 \mathrm{kHz} /{ }^{\circ} \mathrm{C}$;

- cavité tout laiton : $\Delta f / \Delta \theta=-170 \mathrm{kHz} /{ }^{\circ} \mathrm{C}$.

Le gain en stabilité, entre $0^{\circ} \mathrm{C}$ et $100^{\circ} \mathrm{C}$, est donc d'un facteur 6 par rapport à une cavité tout invar et d'un facteur 140 par rapport à une cavité tout laiton.

Pour que la compensation agisse il est nécessaire que la cavité soit à une température bien homogène. Nous utilisons une enceinte à température contrôlée par ventilation d'air chaud ou d'un agent réfrigérant. Il est possible de faire des mesures lors d'une dérive programmée de la température si le taux de variation est inférieur à $0,5^{\circ} \mathrm{C} / \mathrm{mn}$.

A titre d'exemple cette cavité nous a permis [2] de mesurer, sur des substrats d'alumine, le taux de variation de la permittivité avec la température. Pour la partie réelle

$$
\frac{\Delta \varepsilon^{\prime}}{\Delta \theta}=1 \times 10^{-3} \text { à } 1,3 \times 10^{-3}\left({ }^{\circ} \mathrm{C}\right)^{-1}
$$


selon la pureté, et pour la tangente de pertes

$$
\frac{\Delta \operatorname{tg} \theta}{\Delta \theta}=0,8 \times 10^{-6} \text { à } 2,7 \times 10^{-6}\left({ }^{\circ} \mathrm{C}\right)^{-1} .
$$

3. Cavité double. - Cette cavité a été construite en vue d'études de bâtonnets cylindriques de corps ferroélectriques. Du fait des valeurs élevées de $\varepsilon^{\prime}$ et $\operatorname{tg} \delta$ il n'est possible d'introduire dans un maximum de champ électrique qu'un échantillon très fin, dont la réalisation mécanique est très délicate.

Il est donc intéressant d'étudier une disposition où un échantillon de diamètre raisonable (1 à $2 \mathrm{~mm}$ ) se trouve placé dans un minimum de champ électrique. C'est ce qui explique le choix d'un mode TM 120 , l'échantillon étant disposé suivant l'axe de la cavité cylindrique. Pour ce mode, la fréquence de résonance ne dépend que du diamètre de la cavité. Il n'est donc plus possible de réaliser une compensation par le jeu des dilatations différentielles. Nous avons utilisé un dispositif différent qui se distingue par l'utilisation d'une cavité double. Les deux cavités de dimensions identiques sont creusées de façon symétrique dans le même bloc de métal (Fig. 2). L'une des cavités reste

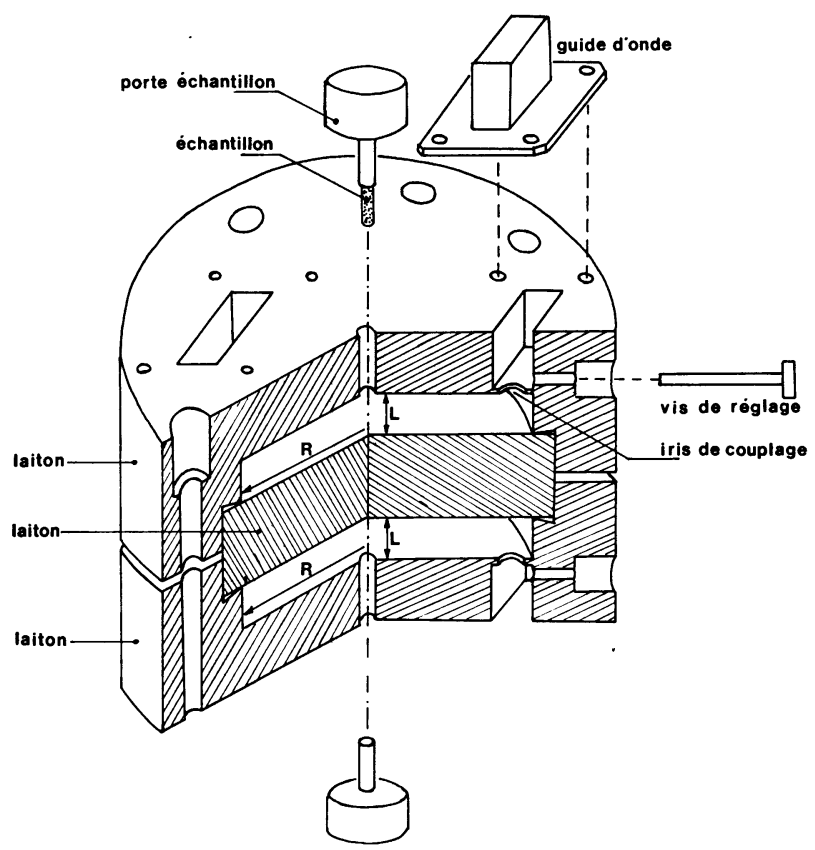

FIG. 2. - Vue générale de la cavité double (Mode TM 120).

[General view of the double cavity (Mode TM 120)]

vide et sert de référence que l'on peut comparer en permanence à la cavité contenant l'échantillon. La comparaison des fréquences propres s'obtient en réalisant un battement entre les générateurs qui entretiennent les oscillations des cavités. Il est alors possible de mesurer les variations en fréquence de l'une des cavités par rapport à l'autre en utilisant un fréquencemètre électronique $0-50 \mathrm{MHz}$, bien moins onéreux qu'un fréquencemètre permettant une mesure directe des fréquences propres des cavités. En contrepartie il faut disposer de deux générateurs hyperfréquences (Fig. 3).

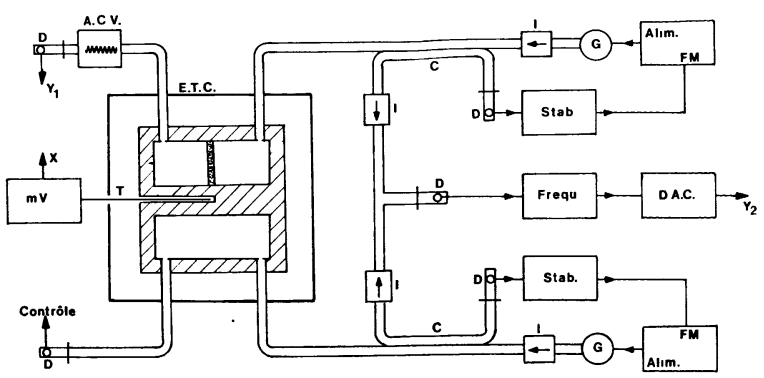

Fig. 3. - Dispositif expérimental utilisant la cavité double ; G : Générateur hyperfréquence ; I : Isolateur ; Alim. : Alimentation du générateur avec prise FM (commande de la fréquence par une tension) ; C : Coupleur ; D : Détecteur ; Stab. : Stabilisateur de fréquence $; \mathrm{mV}:$ millivoltmètre ; Fréqu. : Fréquencemètre numérique ; T : Thermocouple ; D. A. C. : Convertisseur Digital-Analogique; A. C. V.: Atténuateur Calibré Variable ; E. T. C. : Enceinte à température contrôlée ; $X, Y_{1}$, $\mathrm{Y}_{2}$ : Voies d'enregistrement.

[Experimental equipment using the double cavity : G : Microwave Generator ; I : Isolator ; Alim. : power supply with FM entry (frequency control by a voltage) ; C : Coupler ; D : Detector ; Stab. : frequency stabilizer ; $\mathrm{mY}$ : Millivoltmeter ; Frequ. : Frequency counter ; T : Thermo-couple ; D. A. C. : Digital Analog Converter ; A. C. V. : Calibrated Variable Attenuator ; E. T. C. : Controlled Temperature Chamber; $\mathrm{X}, \mathrm{Y}_{1}, \mathrm{Y}_{2}$ : Recording channels.]

Il est avantageux d'utiliser un asservissement [4] maintenant automatiquement l'accord entre le générateur hyperfréquence et la cavité qu'il alimente.

Dans notre réalisation chaque cavité est un cylindre de diamètre $80 \mathrm{~mm}$, de hauteur $10 \mathrm{~mm}$, où le mode TM 120 résonne au voisinage de $8362 \mathrm{MHz}$. L'ensemble est en laiton qui permet un usinage et un polissage aisés. Les couplages sont assurés par des iris disposés sur la face plane de chaque cavité. Des vis d'adaptation permettent de faire varier le coefficient de couplage.

Pour une cavité, considérée isolément, la dérive mesurée de la fréquence propre avec la température est de $-150 \mathrm{kHz} /{ }^{\circ} \mathrm{C}$, valeur en très bon accord avec celle que l'on peut calculer à partir du coefficient de dilatation du laiton. Nous avons relevé expérimentalement une variation linéaire de fréquence dans le domaine exploré de $-50^{\circ} \mathrm{C}$ à $+150^{\circ} \mathrm{C}$. Dans ce même domaine de températures la dérive en fréquence du battement (différence des fréquences propres des deux cavités) est inférieure à $1 \mathrm{kHz} /{ }^{\circ} \mathrm{C}$, ce qui nous permet de la négliger. Rien n'interdit de construire une telle cavité double en invar, ce qui permettrait, pour des dissymétries mécaniques ou thermiques données, de réduire encore par un facteur 20 la dérive du battement.

A titre d'exemple nous présentons les résultats d'une expérience portant sur un échantillon cylindrique (diamètre 1,46 mm) monocristallin, ferroélectrique de Triglycine sulfate (TGS) de formule chimique 


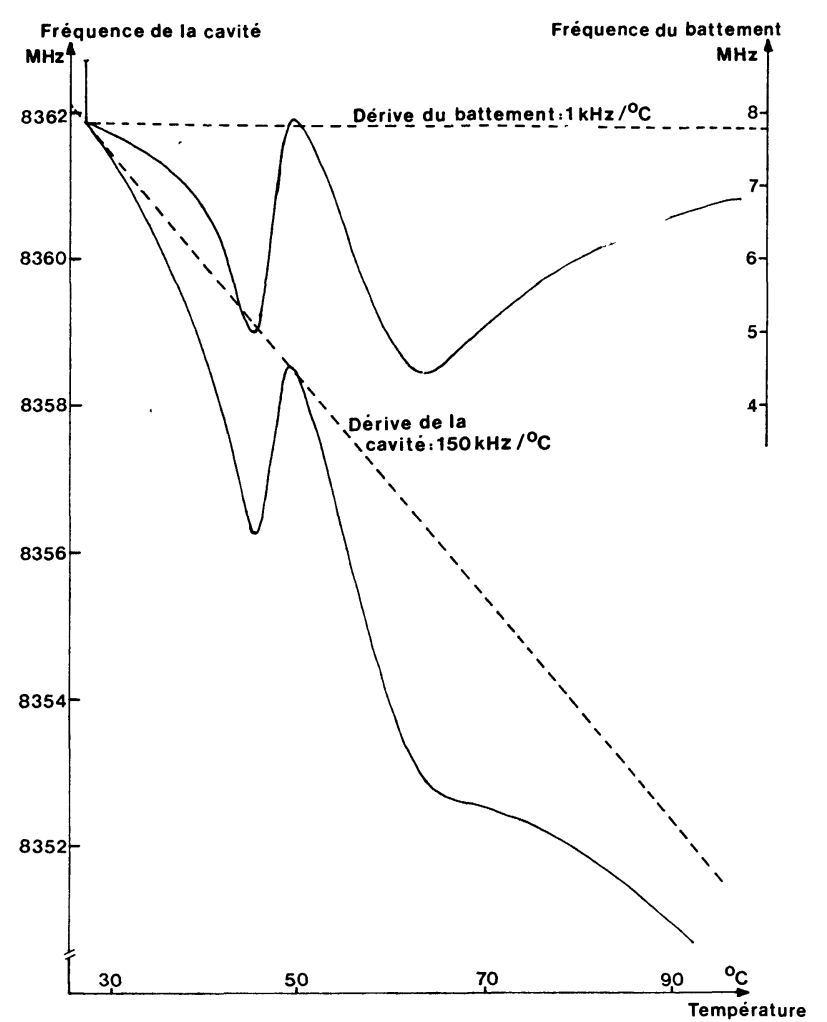

Fig. 4. - Comparaison des résultats obtenus, lors d'une expérience sur TGS, avec une cavité simple, et avec la cavité double réalisant la correction de dilatation.

[Comparison between the curves recorded, in an experiment on TGS, with a single cavity, and with the double cavity with the expansion correction.]
$\left(\mathrm{NH}_{2} \mathrm{CH}_{2} \mathrm{COOH}\right)_{4}, \mathrm{H}_{2} \mathrm{SO}_{3}$. L'axe polaire du cristal est parallèle au champ électrique régnant dans la cavité qui contient l'échantillon.

Les courbes de la figure 4 permettent de comparer la variation, avec la température, de la fréquence propre de la cavité contenant l'échantillon, et la variation de fréquence du battement. Nous voyons que cette dernière courbe réalise automatiquement, et avec une précision suffisante, la correction de dilatation. L'interprétation de cette courbe par la variation avec la température des paramètres $\varepsilon^{\prime}$ et $\operatorname{tg} \delta$ de TGS fera l'objet d'un prochain article.

4. Conclusion. - Nous avons présenté deux procédés, que nous avons expérimentés, qui permettent de réaliser automatiquement, et avec une précision satisfaisante la correction de dilatation lors d'études utilisant des cavités résonnantes soumises à des variations de température.

Le premier procédé (cavité compensée) n'est pas applicable à tous les modes mais sa mise en œuvre est très simple. Le second (cavité double) est applicable à tous les modes et toutes les géométries de cavité, mais il nécessite un doublement de l'appareillage. Il faut toutefois remarquer que les variations de fréquence sont lues sur un simple fréquencemètre 0-50 $\mathrm{MHz}$.

Les performances obtenues sont comparables pour les.deux systèmes, la dérive résiduelle étant de l'ordre de $1 \mathrm{kHz} /{ }^{\circ} \mathrm{C}$ dans une gamme de température allant de $-50^{\circ} \mathrm{C}$ à $+100^{\circ} \mathrm{C}$.

\section{Bibliographie}

[1] Garault, Y., Guillon, F., Jallagéas, H., Com. Organ. Coll. Int. Electron. Mes. (Paris) 1975, p. 171-182.

[2] Vosgien, G., Thèse $3^{\text {e }}$ Cycle Université de Dijon (1976).
[3] Bleaney, B., Loubser, J. H. N., Penrose, R. P., Proc. Phys. Soc. 59 (1947) 185.

[4] Miller, N. D. J., J. Phys. E 9 (1976) 344. 